\title{
Translation, Cross-cultural Adaptation and Validation of the Malay Version of the Maslach Burnout Inventory (MBI) in Malaysia
}

\author{
Won Sun Chen ${ }^{1}$, Jamaiyah Haniff ${ }^{2}$, Ching Sin Siau ${ }^{3}$, Wymen Seet ${ }^{2}$, Sit Fong Loh ${ }^{4}$, Mohd Hadzrul Abd Jamil ${ }^{3}$, Nadian \\ $\mathrm{Sa}^{\prime} \mathrm{at}^{2}$, Nurakmal Baharum ${ }^{2}$ \\ ${ }^{1}$ Monash University Malaysia, Malaysia \\ ${ }^{2}$ National Clinical Research Centre, Ministry of Health, Kuala Lumpur, Malaysia \\ ${ }^{3}$ SEGi University, Malaysia \\ ${ }^{4}$ Turning Point Integrated Wellness Sdn Bhd, Malaysia \\ Correspondence: Won Sun Chen, Monash University Malaysia, Malaysia
}

Received: December 30, 2013 Accepted: January 19, 2014 Available online: February 13, 2014

doi:10.11114/ijsss.v2i2.309 URL: http://dx.doi.org/10.11114/ijsss.v2i2.309

\begin{abstract}
The Maslach Burnout Inventory (MBI) has been widely used in research for more than 2 decades and is recognized as the leading measure of burnout. Malaysia is a multi-ethnic country and English is regarded as the second language. Therefore, it is essential to have a culturally acceptable translated Malay version of MBI, which can easily be understood by Malaysians, in order to study the burnout level in our population. Hence, the objectives of this study are to translate, cross-culturally adapt and validate specifically the Malay versions of the Maslach Burnout Inventory Health Services Survey (MBI-HSS), Maslach Burnout Inventory - Educators Survey (MBI-ES) and Maslach Burnout Inventory - General Survey (MBI-GS) in Malaysia. Intraclass correlation was used to examine the test-retest reliability of the Malay versions of the MBI-HSS, MBI-ES and MBI-GS, while Cronbach's alpha was used to assess the internal consistency of the subscales and the instruments as a whole. Exploratory factor analysis involving the principle component analysis extraction and varimax rotation were used to investigate the construct validity of the instruments. Overall, high intraclass correlation and Cronbach's alpha values were achieved in the Malay versions of the MBI-HSS, MBI-ES and MBI-GS. The eigenvalue in factor analysis revealed all items in the Malay versions of the MBI-HSS, MBI-ES and MBI-GS can be grouped into 3 components, which were very similar to the original English versions. In conclusion, the findings from this study had demonstrated the Malay versions of the MBI-HSS, MBI-ES and MBI-GS were valid and appropriate to be used in Malaysia.
\end{abstract}

Keywords: Maslach Burnout Inventory; Malaysia; psychometric properties; reliability validity

\section{Introduction}

Burnout can be described as the smothering of a fire. This implies that the fire cannot continue to burn brightly unless the resources are replenished. The concept of "burnout" first emerged in 1970s in the United States and it was designated as a progressive emotional depletion, feeling unsuccessful and being worn out (Freudenberger, 1974). Burnout can also be defined as a gradual loss of idealism, energy and purpose (Edelwich \& Brodsky, 1980). Later, "burnout" was defined as a state of exhaustion from physical, mental and emotional aspects (Pines \& Aronson, 1981), or a coping mechanism to manage with stressful and demanding working conditions without recognition (Sarros \& Densten, 1989). The effects of burnout are detrimental, which can ultimately threaten one's job satisfaction, relationships and health (Maslach, 2003a). Lin (2003) claimed that various factors such as job demands, including workload and workplace stress, were the direct cause of emotional exhaustion and depersonalization.

Over the past 35 years, the concept of burnout was used extensively in the United States and later spread into many countries. This phenomenon creates a notable global significance. For example, a study conducted in 1997 revealed the burnout levels for nurses in both United States and the Philippine were influenced by social work environment (Turnipseed \& Turnipseed, 1997). In another study, it was discovered that burnout was prevalent in Jewish and Arab Israelis (Pines, 2003). The concept of burnout was later introduced in Western Europe in the 1980s. From the mid-1990s onwards, the concept of burnout was studied in Eastern Europe, Asia, Middle East, Latin America, Australia and New Zealand (Schaufeli, Leiter \& Maslach, 2009). Kulkarni (2006) in his recent study suggested that burnout issues in the 
developing countries can be greatly influenced by globalization and privatization. This presumably due to with an increased demands of learning new skills and knowledge, society needs to adopt new technology, the pressure to increase productivity and also time pressure.

Despite the fact that "burnout" was studied globally, the definition of burnout varied across languages in many countries, and "burnout" was perceived differently depending on the local social context. For instances, countries like Sweden and the Netherlands recognized burnout as a medical diagnosis where medical consultation and compensation of treatment were possible (Schaufeli et al., 2009).

It is important for organizations to realize that the working environment could potentially be a contributor to the mental ill health and well-being of an employee. This is mainly because mental ill health has been reported as the second highest work related illness after musculoskeletal disorder (Glozier, 2002). Interestingly, burnout is not a recognized disorder in the Diagnostic and Statistical Manual of Mental Disorder (DSM), which is the common language and standard criteria for the classification of mental disorders published by the American Psychiatric Association (Kraft, 2006). On the other hand, burnout is a recognized disorder in the International Statistical Classification of Diseases and Related Health Problems (ICD-10), which is a coding of diseases and signs, symptoms, abnormal findings, complaints, social circumstances and external causes of injury or diseases, as classified by the World Health Organization (WHO) (ICD-10, 1994).

Recently, many organizations in Malaysia have started to place more prominence on the workplace conditions that could possibly improve job satisfaction and also reduce burnout among employees. The basic reason for this initiative is the perception of global shifts in the internal structures and employment practices in the international organizations (Noordin et. al, 2013). On the other hand, cultural differences and the competitive nature in the labor force in Malaysia are potential factors that could burden the employees. Unfortunately, there is no unified definition related to job satisfaction and burnout in Malaysian context, in which most knowledge was originated from individual experiences, opinions of scholars and corridor conversations (Noordin et. al, 2013).

Malaysia's aim is to emerge as a developed nation by 2020. This scenario leads to an increase in the labor force competitiveness level which can undoubtedly direct to more burnout cases. Therefore, it is vital to have instruments in the national language of Malaysia (Bahasa Malaysia), which can help to screen and measure the burnout levels. Hence, this study aims at translating, cross culturally adapting and validating the Malay versions of the Maslach Burnout Inventory - Health Services Survey (MBI-HSS), Maslach Burnout Inventory - Educators Survey (MBI-ES) and Maslach Burnout Inventory - General Survey (MBI-GS) using a larger sample. Specifically, this study consists of 2 parts; Part I with an objective to translate and cross-culturally adapt the Malay versions of the MBI-HSS, MBI-ES and MBI-GS, while Part II was to validate the Malay versions of the MBI-HSS, MBI-ES and MBI-GS. The pilot study of these instruments revealed acceptable psychometric properties (Chen et al., 2014).

Study Materials

Maslach Burnout Inventory (MBI)

Although the concept of burnout aroused a huge interests in the early years, however, only limited information was available in guiding the empirical research. A group of researchers took the initiatives to develop and establish a standardized measurement of an individual's experience of burnout. Today, the Maslach Burnout Inventory (MBI) has been broadly acknowledged as the leading measure of burnout. Generally, the MBI addresses three subscales; 1) emotional exhaustion which measures feelings of being emotionally overextended and exhausted due to work, 2) depersonalization which measures the unfeeling and impersonal response towards one's service and care treatment, and 3) personal accomplishment which measures one's successful achievement and competency at work. This instrument was further been enhanced to measure the perception of burnout from three defined groups on their job as well as the people whom they closely interact with. These instruments are known as MBI - Human Services Survey (MBI-HSS), MBI Educators Survey (MBI-ES), and MBI - General Survey (MBI-GS). All the items in these instruments were scored on a seven-point Likert scale from 0 (never) to 6 (everyday) (Leiter \& Maslach, 2005a; Leiter \& Maslach, 2005b; Maslach, 2003b; Maslach, Jackson \& Leiter, 1996; Maslach \& Leiter, 1997; Maslach \& Leiter, 2008; Maslach et al., 2009).

\section{Maslach Burnout Inventory - Human Services Survey (MBI-HSS)}

Human services professionals, such as counselors or nurses spend considerable amount of time interacting with others. The face-to-face staff-client interaction focuses on the client's psychological, social or physical problems. The staffs who continuously deal with client's anger, frustrating or embarrassing emotions may find their experience draining and exhausting, which can eventually lead to burnout. Therefore, MBI-HSS was created specifically to measure burnout as an occupational issue for people who have direct interactions with clients. MBI-HSS consists of 22 items from 3 subscales, namely 1) emotional exhaustion, 2) personal accomplishment and 3) depersonalization (Maslach et al., 1996).

\section{Maslach Burnout Inventory - Educators Survey (MBI-ES)}

Teaching professionals are always been subjected to higher pressure by community to expand their roles beyond 
educations. Since teachers are usually the first point of contact for students, therefore, teachers are expected to have frequent interactions or consultations with students, to address students' social problems (such as family problems, relationship issues, addictions to drug, alcohol-related issues) and also to contribute to students' moral and ethical development. All these demanding expectations can gradually lead to a feeling of demotivation towards their jobs and may also cause teachers to leave their teaching profession. MBI-ES was designed as a measurement of burnout among teaching professionals. It has a total of 22 items from 3 subscales, namely 1) emotional exhaustion, 2) personal accomplishment and 3) depersonalization (Maslach et al., 1996).

\section{Maslach Burnout Inventory - General Survey (MBI-GS)}

MBI-GS is a modified version of MBI-HSS designed specifically to measure burnout as an occupational issue for professions other than public human services. The MBI-GS measured burnout from the perspective of one's performance at work in general. It comprised 16 items from 3 subscales, namely, 1) professional efficacy, 2) exhaustion and 3) cynicism (Maslach et al., 1996).

\section{Methods}

Part I: Translation and cross culturally adaptation process

\section{Stage 1: Forward translation process}

Permission was obtained from Mind Garden, Inc. to translate the English versions of the MBI-HSS, MBI-ES and MBI-GS into Malay. In the forward translation process, a group of medical experts and 2 independent bilingual professional translators translated the English versions of the MBI-HSS, MBI-ES and MBI-GS into Malay. Each translator provided a written report of the translated draft. These reports were reviewed by the researchers when harmonizing the pre-final Malay version of the instruments.

\section{Stage 2: Backward translation process}

The pre-final version of the Malay instruments was then back translated into English by a group of medical experts and 2 independent bilingual professional translators. This is known as the backward translation process. New English version of the instruments was developed by referring to the comments in the written reports provided by the translators. The aim of the backward translation process was to compare each item of the new English version of the instruments with the original English version of MBI.

\section{State 3: Consensus meeting}

A consensus meeting involving the translators, medical experts and a multi-disciplinary researcher team was held to review, reconcile and harmonize the Malay version of the instruments (Chen, 2009).

\section{Stage 4: Pre-testing the translated instruments}

Three different focus groups, comprised 10 respondents for MBI-HSS, 10 respondents for MBI-ES and 10 respondents for MBI-GS, were recruited to pre-test the Malay versions of the MBI-HSS, MBI-ES and MBI-GS. Each focus group was chaired by a moderator. All respondents in each focus group were asked to answer one instrument in both English and Malay versions and their comments with regard to the clarity of language used were documented accordingly.

\section{Stage 5: Final consensus meeting}

A consensus meeting among the multi-disciplinary researcher team was held again to review and discuss the comments documented from the respondents in focus groups. Consensus among the team was reached in revising and finalizing the Malay versions of the MBI-HSS, MBI-ES and MBI-GS.

\section{Part II: Validation process}

\section{Sample size justification}

It was suggested that a minimum of 5 respondents per item (Gorsuch, 1983) or 10 respondents per item (Everitt, 1975) necessary for any questionnaire. Assuming 10 respondents were required for each item per questionnaire, thus, MBI-HSS and MBI-ES required 220 respondents each while only 190 respondents were required for MBI-GS. Therefore, a total of 630 respondents were required for the validation study.

\section{Selection of respondents}

The respondents for MBI-HSS were mainly nurses from Hospital Kuala Lumpur whereas instructors from few private universities located in the area of Klang Valley were selected as the respondents for MBI-ES. All respondents in the MBI-GS were general administrative support staff from both private and public sectors. Generally, all respondents were selected conveniently around the area of Klang Valley during the period of January-August 2013. The selected respondents were at least 21 years old and proficient in Malay language. 


\section{Study design and procedure}

This was a cross-sectional study. All respondents were asked to sign the informed consent letter prior to the commencement of this study. Each respondent spent approximately 30 minutes to complete a demographic form along with a Malay MBI instrument depending on the respondent's professional background. After a 2-week interval, similar respondents were approached for their response on the same Malay MBI instrument.

\section{Reliability analysis}

The test-retest reliability analysis was used to examine the reliability of the Malay versions of the MBI-HSS, MBI-ES and MBI-GS after a 2-week interval (Chen, 2009). This type of reliability was evaluated by an intraclass correlation (ICC), which was computed based on a single rate using the one way analysis of variance (ANOVA) model (Streiner \& Norman, 1995). By convention, ICC value of 0.70 was regarded as an acceptable reliability coefficient (Maurice, Staquet \& Peter, 1998; Nunnally \& Bernstien, 1994). On the other hand, Cronbach's alpha was used to assess the internal consistency of the subscales as well as the instrument as a whole. In practice, Cronbach's alpha of at least 0.70 was desirable (Bland \& Altman, 1997; Cronbach, 1951; DeVellis, 2003; Nunnally \& Bernstien, 1994).

Validity analysis

Validity analysis was used to ensure the underlying scale measured what it was supposed to measure. Exploratory factor analysis involving the principle component analysis extraction and varimax rotation was used to assess the construct validity of the Malay versions of the MBI-HSS, MBI-ES and MBI-GS (Chen, 2009).

\section{Results}

Demographic background of respondents

Table 1. Demographic background of respondents

\begin{tabular}{|c|c|c|c|}
\hline Demographic & $\begin{array}{c}\text { Malay MBI-HSS } \\
\mathrm{n}=220\end{array}$ & $\begin{array}{c}\text { Malay MBI-ES } \\
n=229\end{array}$ & $\begin{array}{c}\text { Malay MBI-GS } \\
n=123\end{array}$ \\
\hline \multicolumn{4}{|l|}{ Age in years } \\
\hline Mean \pm SD & $33.1 \pm 9.7$ & $36.7 \pm 8.8$ & $33.1 \pm 7.9$ \\
\hline Median (min, max) & $30.0(21,62)$ & $36.0(21,66)$ & $31.0(22,61)$ \\
\hline \multicolumn{4}{|l|}{ Gender $(\mathrm{n}, \%)$} \\
\hline Male & $53(24.1)$ & $71(31.0)$ & $32(26.2)$ \\
\hline Female & $167(75.9)$ & $158 \quad(69.0)$ & $90(73.8)$ \\
\hline \multicolumn{4}{|l|}{ Race $(n, \%)$} \\
\hline Malay & $199(90.5)$ & $70(30.7)$ & $59(48.0)$ \\
\hline Chinese & $5(2.3)$ & $112(49.1)$ & $43(35.0)$ \\
\hline Indian & $13(5.9)$ & $42(18.4)$ & $18(14.6)$ \\
\hline Others & $3(1.4)$ & $4(1.8)$ & $3(2.4)$ \\
\hline \multicolumn{4}{|l|}{ Marital status (n, \%) } \\
\hline Single & $79(35.9)$ & $74(32.5)$ & $60(48.8)$ \\
\hline Married & $139(63.2)$ & $146(64.0)$ & $59(48.0)$ \\
\hline Divorced & $0(0)$ & $7(3.1)$ & $1(0.8)$ \\
\hline Widowed & $2(0.9)$ & $1(0.4)$ & $3(2.4)$ \\
\hline \multicolumn{4}{|l|}{ Educational (n, \%) } \\
\hline Primary school & $1(0.5)$ & $0(0)$ & $0(0)$ \\
\hline Secondary school & $64(29.5)$ & $1(0.4)$ & $4(3.3)$ \\
\hline College/university & $148(68.2)$ & $219(95.6)$ & $112(93.3)$ \\
\hline None & $1(0.5)$ & $1(0.4)$ & $2(1.7)$ \\
\hline Others & $3(1.4)$ & $8(3.5)$ & $2(1.7)$ \\
\hline \multicolumn{4}{|l|}{ Income (n, \%) } \\
\hline$<$ RM2000 & $77(35.0)$ & $3(1.4)$ & $24(21.4)$ \\
\hline RM2000-RM4000 & $122(55.5)$ & $103(47.0)$ & $67(59.8)$ \\
\hline RM4000-RM6000 & $10(4.5)$ & $85(38.8)$ & $10(8.9)$ \\
\hline RM6000-RM8000 & $6(2.7)$ & $18(8.2)$ & $7(6.3)$ \\
\hline RM8000-RM10000 & $2(0.9)$ & $5(2.3)$ & $0(0)$ \\
\hline$>$ RM10000 & $3(1.4)$ & $5(2.3)$ & $4(3.6)$ \\
\hline \multicolumn{4}{|l|}{ Occupation (n, \%) } \\
\hline Employer & $8(3.7)$ & $2(0.9)$ & $5(4.1)$ \\
\hline Professional & $93(43.3)$ & $212(93.8)$ & $39(32.2)$ \\
\hline Technical \& semi-professional & $20(9.3)$ & $6(2.7)$ & $3(2.5)$ \\
\hline Administrative support staff & $37(17.2)$ & $3(1.3)$ & $59(48.8)$ \\
\hline Sales and services & $2(0.9)$ & $0(0)$ & $6(5.0)$ \\
\hline $\begin{array}{l}\text { Police, army and uniform } \\
\text { professionals }\end{array}$ & $7(3.3)$ & $0(0)$ & $4(3.3)$ \\
\hline Others & $48(22.3)$ & $3(1.3)$ & $5(4.1)$ \\
\hline
\end{tabular}


Table 1 revealed the minimum required sample size was achieved for both MBI-HSS and MBI-ES, but a slightly lower size was obtained for MBI-GS due to resources constraint.

For MBI-HSS, the respondents were aged between 21 to 62 years old with a median age of 30 years old. Majority of the respondents in this group were female (75.9\%), Malay (90.5\%), married (63.2\%), had tertiary educational background $(68.2 \%)$ and working as professionals (43.3\%) with monthly income of RM2000 to RM4000.

The respondents in the MBI-ES group were aged between 21 to 66 years old with a median age of 36 years old. This group predominantly comprised female $(69.0 \%)$, Chinese $(49.1 \%)$, married $(64.0 \%)$, had tertiary educational background (95.6\%) and working as professionals (93.8\%) with monthly income of RM2000 to RM4000.

Among the respondents in the MBI-GS group, the age was ranged from 22 to 61 years old with a median age of 31 years old. Most of the respondents in this group were female $(73.8 \%)$, Malay $(48.0 \%)$, single $(48.8 \%)$, had tertiary educational background (93.3\%) and working as administrative support staff (48.8\%) with monthly income of RM2000 to RM4000.

Table 2. Intraclass correlation (ICC) and Cronbach's alpha

\begin{tabular}{|c|c|c|c|c|}
\hline Type of MBI & Sub-scale & ICC & $\begin{array}{l}\text { Cronbach's } \\
\text { alpha }\end{array}$ & $\begin{array}{c}\text { Overall Cronbach's } \\
\text { alpha }\end{array}$ \\
\hline \multirow{3}{*}{$\begin{array}{l}\text { Malay } \\
\text { MBI-HSS }\end{array}$} & Emotional exhaustion (EE) & 0.847 & 0.849 & \multirow[t]{3}{*}{0.803} \\
\hline & Depersonalization (DEP) & 0.774 & 0.773 & \\
\hline & Personal achievement (PA) & 0.726 & 0.732 & \\
\hline \multirow{3}{*}{$\begin{array}{l}\text { Malay } \\
\text { MBI-ES }\end{array}$} & Emotional exhaustion (EE) & 0.913 & 0.917 & \multirow[t]{3}{*}{0.788} \\
\hline & Depersonalization (DEP) & 0.780 & 0.781 & \\
\hline & Personal achievement (PA) & 0.788 & 0.787 & \\
\hline \multirow{3}{*}{$\begin{array}{l}\text { Malay } \\
\text { MBI-GS }\end{array}$} & Exhaustion (EX) & 0.797 & 0.798 & \multirow[t]{3}{*}{0.801} \\
\hline & Cynicism (CY) & 0.876 & 0.875 & \\
\hline & Personal efficiency (PE) & 0.876 & 0.878 & \\
\hline
\end{tabular}

Reliability analysis

For MBI-HSS, both ICC and Cronbach's alpha values were beyond the desirable level. Specifically, the ICC was 0.847, 0.774 , and 0.726 for emotional exhaustion, depersonalization and personal achievement subscales respectively. On the other hand, the Cronbach's alpha value was $0.849,0.773$ and 0.732 for emotional exhaustion, depersonalization and personal achievement subscales accordingly. The overall Cronbach's alpha value was 0.803 .

In MBI-ES, the ICC was $0.913,0.780$ and 0.788 for emotional exhaustion, depersonalization and personal achievement respectively. In addition, the Cronbach's alpha value was 0.917 for emotional exhaustion, 0.781 for depersonalization and 0.787 for personal achievement subscales. The overall Cronbach's alpha value was 0.788 . Both ICC and Cronbach's alpha values were beyond the desirable level.

As for the MBI-GS group, the ICC was found to be 0.797 (exhaustion), 0.876 (cynicism) and 0.876 (personal efficacy) subscales. Analogously, the Cronbach's alpha values were 0.798 for exhaustion, 0.875 for cynicism and 0.878 for personal efficacy subscales. The overall Cronbach's alpha value was 0.801 . Both ICC and Cronbach's alpha values were well above the desirable level.

Validity analysis

Generally, the eigenvalue in factor analysis revealed all items in the Malay versions of the MBI-HSS, MBI-ES and MBI-GS can be grouped into 3 subscales (Table 3).

In MBI-HSS, the results showed that 5 items (item 6,13,14,16 and 20) have deviated from the original subscales. All 5 items except item 14 were swapped between the subscales of emotional exhaustion and depersonalization. On the other hand, item 14 was found to swap between the emotional exhaustion and personal achievement subscales.

For MBI-ES, the rotated loading factors value for 3 items, namely items 11, 12 and 22, were higher in the emotional exhaustion subscale instead of their original subscales, namely depersonalization subscale for items 11 and 22 and personal achievement subscale for item 12 .

In MBI-GS, both items 8 and 9 deviated from their original subscale, cynicism, to exhaustion subscale. 
Table 3. Loading factors from principal component analysis with varimax rotation

\begin{tabular}{|c|c|c|c|c|c|c|c|c|c|}
\hline \multirow[t]{3}{*}{ Items } & \multicolumn{3}{|c|}{ Malay MBI-HSS } & \multicolumn{3}{|c|}{ Malay MBI-ES } & \multicolumn{3}{|c|}{ Malay MBI-GS } \\
\hline & \multicolumn{3}{|c|}{ Component } & \multicolumn{3}{|c|}{ Component } & \multicolumn{3}{|c|}{ Component } \\
\hline & 1 (DP) & $2(\mathrm{PA})$ & $3(\mathrm{EE})$ & $1(\mathrm{EE})$ & $2(\mathrm{PA})$ & 3 (DP) & $1(\mathrm{EX})$ & $2(\mathrm{PE})$ & $3(\mathrm{CY})$ \\
\hline 1 & & & 0.691 & 0.856 & & & 0.799 & & \\
\hline 2 & & & 0.765 & 0.806 & & & 0.877 & & \\
\hline 3 & & & 0.746 & 0.810 & & & 0.824 & & \\
\hline 4 & & 0.550 & & & 0.498 & & 0.857 & & \\
\hline 5 & 0.671 & & & & & 0.644 & & 0.443 & \\
\hline 6 & 0.600 & & 0.321 & 0.588 & & & 0.857 & & \\
\hline 7 & & 0.647 & & & 0.648 & & & 0.787 & \\
\hline 8 & & & 0.691 & 0.864 & & & 0.690 & & 0.302 \\
\hline 9 & & 0.612 & & & 0.629 & & 0.694 & & 0.297 \\
\hline 10 & 0.687 & & & & & 0.729 & & 0.760 & \\
\hline 11 & 0.722 & & & 0.578 & & 0.552 & & 0.792 & \\
\hline 12 & & 0.525 & & -0.556 & 0.520 & & & 0.819 & \\
\hline 13 & 0.774 & & 0.196 & 0.543 & & & & & 0.246 \\
\hline 14 & & 0.374 & 0.138 & 0.391 & & & & & 0.874 \\
\hline 15 & 0.730 & & & & & 0.671 & & & 0.738 \\
\hline 16 & 0.709 & & 0.120 & 0.560 & & & & 0.789 & \\
\hline 17 & & 0.796 & & & 0.714 & & & & \\
\hline 18 & & 0.784 & & & 0.753 & & & & \\
\hline 19 & & 0.716 & & & 0.600 & & & & \\
\hline 20 & 0.710 & & 0.316 & 0.679 & & & & & \\
\hline 21 & & 0.669 & & & 0.644 & & & & \\
\hline 22 & 0.449 & & & 0.431 & & 0.247 & & & \\
\hline
\end{tabular}

\section{Discussion}

The overall findings from this study demonstrated that the Malay versions of the MBI-HSS, MBI-ES and MBI-GS possessed acceptable reliability and validity. By validating these instruments in Malaysia, it shows that the Malay versions are able to measure the burnout levels in our population. We want to encourage more awareness and assessments relevant to burnout to be conducted locally.

Since high value of ICC (>0.80) indicated the effect of measurement error on correlation was minimal (Weir, 2005), therefore, the ICC presented for MBI-HSS (ranging from 0.726 to 0.847 ) clearly indicated negligible measurement error. On the other hand, all Cronbach's alpha values reported for MBI-HSS were well above the desirable value of 0.700 . It was revealed in a recent study that a high value of Cronbach's alpha $(>0.900)$ suggested redundancies and indicated the length of the instrument should be shorten (Tavakol \& Dennick, 2011). Therefore, the overall Cronbach's alpha value of 0.803 indicated the length of the MBI-HSS was just appropriate to be used. The Cronbach's alpha values for all subscales reported for MBI-HSS were very similar to those published in the technical manual for MBI (Maslach et al., 1996).

For MBI-HSS, it was interesting to reveal 4 items (items 6, 13, 16 and 20) out of a total of 22 items deviated from the original subscales. Generally, these 4 items were found to have swapped between the depersonalization and emotional exhaustion subscales due to the words used. In item 6, an in-depth investigation showed the original wording of "working with people all day is really a strain for me" was in the emotional exhaustion subscale, while the Malay wording carried the meaning of "working with people all day is really stressful for me" fell into the depersonalization subscale. Analogously, the English wording of "working with people directly puts too much stress on me" in item 16 was translated into "working with people directly puts too much pressure on me" in item 16. Although the Malay word of "tekanan (pressure)" was a direct translation of the word "strain" and "stress", these words were perceived differently in the Malaysian context. On the other hand, the original wording for item 13 "I feel frustrated by my job" was interpreted differently in the Malay version as "I feel disappointed by my job". In Asian culture, it was not common to hear the phrase "I feel like I'm at the end of my rope" as in item 20. Therefore, the direct translation of "I feel like I am unable to withstand it anymore" caused confusions among the respondents. The perception of item 14 "I feel I'm working too hard on my job" was slightly different for Malaysians. It was perceived as a personal achievement of being able to work hard instead of an emotional exhaustion aspect. 
In MBI-ES, the ICC value of 0.788 to 0.913 clearly indicated negligible measurement error (Weir, 2005). The overall Cronbach's alpha value of 0.788 showed the length of MBI-ES was just appropriate to be used (Tavakol \& Dennick, 2011). The Cronbach's alpha values for all subscales reported in MBI-ES were very similar to those published in the technical manual for MBI (Maslach et al., 1996).

The rotated loading factors for items 11 and 12 in MBI-ES were found to be slightly higher in the emotional exhaustion subscale instead of their original subscales, namely original subscales, depersonalization subscale and personal achievement subscale respectively. The negligible increment in the rotated loading factors will not have enough support to move these items away from their original subscale. On the other hand, it was interesting to note that Malaysians perceived item 22 "I feel students blame me for some of their problems" as emotional exhaustion instead of depersonalization.

The ICC presented in MBI-GS (ranging from 0.797 to 0.876) clearly indicated negligible measurement error (Weir, 2005). The overall Cronbach's alpha value of 0.801 justified the length of MBI-GS was just appropriate to be used (Tavakol \& Dennick, 2011). The Cronbach's alpha values for all subscales reported in MBI-GS were very similar to those published in the technical manual for MBI (Maslach et al., 1996).

Two items, specifically items 8 and 9, in MBI-GS were found to be deviated from their original subscale, cynicism, to exhaustion subscale. In-depth analysis revealed Malaysians perceived item 8 "I've become less interested in my work since I started this job" and item 9 "I have become less enthusiastic about my work" as exhaustion instead of cynicism.

It was reported in recent studies that differential items may be attributed to translation errors, but it was also likely due to differences in cultural knowledge and experience (Elis, 1989). Recent study concluded that Japanese people, whom are the members of Eastern culture, were found to be low in self disclosure in both verbal and non-verbal measures (Miyahara, 2000). In general, emotional moderation was expected to be observed for those in the Eastern cultures compared to Western cultures (Niedenthal, et al., 2006, pgs 314-15). In spite of its deficiencies, high ICC and Cronbach's alpha values were achieved in the Malay versions of the MBI-HSS, MBI-ES and MBI-GS. The pilot study of these instruments also revealed acceptable psychometric properties (Chen et al., 2014).

\section{Conclusion}

In conclusion, this study confirmed that the Malay versions of the MBI-HSS, MBI-ES and MBI-GS have acceptable psychometric properties. They were valid and appropriate to be used to facilitate future studies related to burnout in Malaysia.

\section{Limitations}

Some limitations existed in this study. The respondents for this study were recruited from the urban area of Klang Valley due to logistic constraints. Previous studies (Abel \& Sewell, 1999; Mackie, 2008) indicated that the burnout level was different for those in the rural and urban areas. Therefore, it would be interesting to compare the findings from this study with those in the rural areas. In near future, studies comprising both rural and urban population can be conducted for better review and comparison.

\section{Acknowledgement}

The authors would like to thank the Director General of Health Malaysia for the permission to publish this paper. In addition, we express appreciation to Mind Garden, Inc. for the permission to use the English versions of the MBI-HSS, MBI-ES and MBI-GS. Also, we would like to extend our acknowledgement to A. Reenaa Joys A/P Andas for editing our manuscript. This project was approved by the Medical Research Ethics Committee, Ministry of Health Malaysia and also the SEGi University Ethics Committee. It was fully funded by an Exploratory Research Grant Scheme (ERGS/1/2011/SS/SEGI/03/1) from the Ministry of Higher Education Malaysia.

\section{References}

Abel, M. H., \& Sewell, J. (1999). Stress and burnout in rural and urban secondary school teachers. Journal of Educational Research, 92, 287-293. http://dx.doi.org/10.1080/00220679909597608

Bland, J., \& Altman D. (1997). Statistics notes: Cronbach alpha. BMJ. 314-275. http://dx.doi.org/10.1136/bmj.314.7080.572

Chen W. S. (2009). Questionnaire translation and psychometric properties evaluation. SEGi Review, 2(2), 62-71.

Chen, W. S., Haniff, J., Siau, C. S., Seet, W., Loh, S. F., \& Abd Jamil, M. H. (2014). Pilot study of the Malay Maslach Burnout Inventory and Malay Work-Related Quality of Life Scale in Malaysia. Studies in Asian Social Science, 1(1), 20-26.

Cronbach, L. F. (1951). Coefficient alpha and the internal structure of tests. Psychometricka, 16, 297-334. 
http://dx.doi.org/10.1007/BF02310555

DeVellis, R. (2003). Scale development: theory and applications. Thousand Oaks, CA: Sage.

Edelwich, J., \& Brodsky, A. (1980). Burnout: stages of disillusionment in the helping professions. New York: Human science press.

Elis, B. B. (1989). Differential item functioning: implication for test translation. Journal of Applied Psychology, 74(6), 912-921. http://dx.doi.org/10.1037/0021-9010.74.6.912

Everitt B. S. (1975). Multivariate analysis: The need for data, and other problems. British Journal of Psychiatry, 126, 237-240. http://dx.doi.org/10.1192/bjp.126.3.237

Freudenberger, H. J. (1974). Staff burnout. Journal of social issues, 30, 159-65. http://dx.doi.org/10.1111/j.1540-4560.1974.tb00706.x

Glozier, N. (2002). Mental ill health and fitness for work. Journal of occupational and environmental medicine, 59, 714-720. http://dx.doi.org/10.1136/oem.59.10.714

Gorsuch R. L. (1983). Factor analysis ( $2^{\text {nd }}$ ed.). Hillsdale, NJ: Lawrence Erlbaum Associates.

ICD-10. (1994). International classification of diseases, World Health Organization, Geneva.

Kraft, U. (2006). Burned out: your job is extremely fulfilling. It is also extremely demanding - And you feel overwhelmed. You are not alone. Scientific American Mind, June/July 2006, page 29-33.

Kulkarni, G. K. (2006). Burnout (Editorial). Indian Journal of Occupational and Environmental Medicine, 10, 3-4. http://dx.doi.org/10.4103/0019-5278.22887

Leiter, M. P., \& Maslach, C. (2005a). Banishing Burnout: Six strategies for improving your relationship with work. San Francisco, CA: Jossey-Bass.

Leiter, M. P., \& Maslach, C. (2005b). A mediation model of job burnout. In Antoniou, A. S., \& Cooper, C. L. (Eds.) Research companion to organizational health psychology. Cheltenham, United Kingdom: Elgar Publishing. pp. 544-564. http://dx.doi.org/10.4337/9781845423308.00046

Lin, Y. W. (2013). The causes, consequences, and mediating effects of job burnout among hospital employees in Taiwan. Journal of Hospital Administration, 2(1), 15-27.

Mackie, P. F. E. (2008). Are social workers really burned out? An analysis between rural and urban social workers. Journal of Rural Mental Health, 32(2), 3-18. http://dx.doi.org/10.1037/h0095947

Maslach, C. (2003a). Burnout: The Cost of Caring. Los Altos: Malor Book.

Maslach, C. (2003b). Job burnout: New directions in research and intervention. Current Directions in Psychological Science, 12, 189-192. http://dx.doi.org/10.1111/1467-8721.01258

Maslach, C, \& Leiter, M. P. (1997). The Truth about Burnout: How organizations cause personal stress and what to do about it. San Francisco, CA: Jossey-Bass.

Maslach, C., \& Leiter, M. P. (2008). Early predictors of job burnout and engagement. Journal of Applied Psychology, 93, 498-512. http://dx.doi.org/10.1037/0021-9010.93.3.498

Maslach, C., Jackson, S. E., \& Leiter, M. P. (1996). Maslach Burnout Inventory Manual ( $3^{\text {rd }}$ edition). Menlo Park, CA: Mind Garden.

Maslach, C., Leiter, M. P., \& Schaufeli, W. B. (2009). Measuring burnout. In S. Cartwright \& C. L. Cooper (Eds.) The Oxford handbook of organizational well-being. Oxford, United Kingdom: Oxford University Press. Pp. 86-108.

Maurice, J., Staquet, R. H. D., \& Peter, M. F. (1998). Quality of life assessment in clinical trials: methods and practice. New York: Oxford University Press.

Miyahara, A. (2000). Toward Theorizing Japanese Interpersonal Communication Competence from a Non-Western Perspective. American Communication Journal, 3(3), 279

Niedenthal, P. M., Krauth-Gruber, S., \& Francois, R. (2006) Psychology of Emotion Interpersonal, Experimental, and Cognitive Approaches (pp. 5, 305-342) New York, NY: Psychology Press.

Noordin, F., Wan Shukran, S. S., Abdul Hamid, S. M., \& Hamali, J. (2013). Burnout and academics: a case of a public university in Malaysia. $4^{\text {th }}$ International Conference on Business and Economic Research $4^{\text {th }}$ ICBER 2013) Proceeding, 4-5 March 2013, Bandung, Indonesia.

Nunnally, J. C., \& Bernstien, I. H. (1994). Psychometric theory 3. New York: McGraw-Hill. 
Pines, A., \& Aronson, E. (1981). From tedium to personal growth. New York: Free press.

Pines, A. M. (2003). Occupational burnout: a cross-cultural Israeli Jewish-Arab perspective and its implications for career counseling. Career Development International, 8, 97-106. http://dx.doi.org/10.1108/13620430310465516

Sarros, J. C., \& Densten, I. L. (1989). Undergraduate students stress and coping strategies. Higher education research and development, 8, 47-57. http://dx.doi.org/10.1080/0729436890080105

Schaufeli, W. B., Leiter, M. P., \& Maslach, C. (2009). Burnout: 35 years of research and practice. Career Development International, 14(3), 204-220. http://dx.doi.org/10.1108/13620430910966406

Streiner, D. L., \& Norman, G. R. (1995). Health measurement scales: a practical guide to their development and use. New York: Oxford University Press

Tavakol, M., \& Dennick, R. (2011). Making sense of Cronbach's alpha. International Journal of Medical Education, 2, 53-55. http://dx.doi.org/10.5116/ijme.4dfb.8dfd

Turnipseed, D. L., \& Turnipseed, P. H. (1997). A bi-cultural analysis of the costs of caring: nursing burnout in the United States and the Philippines. Career Development International, 2, $180-188$. http://dx.doi.org/10.1108/13620439710173670

Weir, J. P. (2005). Quantifying test-retest reliability using the intraclass correlation coefficient and the SEM. Journal of Strength and conditioning research, 19(1), 231-240.

\section{(cc) $\mathrm{Br}$}

This work is licensed under a Creative Commons Attribution 3.0 License. 